\title{
Obtención de capas de grafeno a través de la exfoliación mecánica del grafito
}

Obtaining graphene layers through the mechanical exfoliation of graphite

\author{
Edmundo Guacho Guado. ${ }^{1}$, Celin Padilla Padilla. ${ }^{2}$, Luis Buenaño Moyano. ${ }^{3}$ \& Bolívar \\ Cuaical Angulo. ${ }^{4}$
}

Recibido: 28-10-2018 / Revisado: 29-11-2018 /Aceptado: 18-12-2018/ Publicado: 05-01-2019

\begin{abstract}
.
DOI: https://doi.org/10.33262/cienciadigital.v3i1.294

Graphene contains a flat laminar structure which is formed by hexagonal rings, thanks to which it is an ultralight material. Through alloys this material can contribute to improve the properties of other materials since a perfect graphene gram could only be broken with a diamond. Graphene highlighted its importance in the year 2004 since then studies are made about this element that could be applied in all areas of engineering in order to fully enter the market and make improvements in most products. This article aims to demonstrate the structure and properties of graphene in order to reach an alloy with a metal and verify its strength. There are several methods to verify the structure of graphene, which are based on graphite. In this research, minimum amounts of graphite were used to detach a sample layer by layer until reaching a very thin layer in such a way that it can be analyzed through a microscope. In addition, it seeks to venture and find elements with which alloys can be made, because graphene is considered one of the elements of the future as it could replace several metals to be able to form light structures five hundred times more resistant.
\end{abstract}

Keywords: Graphene, Laminar Structure, Hexagonal Rings, Graphite

\footnotetext{
${ }^{1}$ Escuela Superior Politécnica de Chimborazo, Grupo de Investigación SAPIA, Chimborazo, Ecuador, edmundo.guacho@espoch.edu.ec

${ }^{2}$ Escuela Superior Politécnica de Chimborazo, Grupo de Investigación SAPIA, Chimborazo, Ecuador, bcuaical@espoch.edu.ec

${ }^{3}$ Escuela Superior Politécnica de Chimborazo, Grupo de Investigación SAPIA a, Chimborazo, Ecuador, lfbuenanio@espoch.edu.ec

${ }^{4}$ Escuela Superior Politécnica de Chimborazo, Grupo de Investigación SAPIA, Chimborazo, Ecuador, bcuaical@espoch.edu.ec
} 


\section{Resumen.}

El grafeno contiene una estructura laminar plana la cual está formada por anillos hexagonales, gracias a lo cual logra ser un material ultraligero. Mediante aleaciones este material puede contribuir a mejorar las propiedades de otros materiales ya que un gramo perfecto de grafeno solo se podría romper con un diamante. El grafeno resaltó su importancia en el año 2004 desde entonces se realizan estudios acerca de este elemento que podría aplicarse en todos los ámbitos de la ingeniería con el fin de adentrarse por completo en el mercado y hacer mejoras en la mayor parte de productos. Este articulo pretende demostrar la estructura y las propiedades del grafeno para poder llegar a una aleación con un metal y verificar su resistencia. Existen varios métodos para verificar la estructura del grafeno, los cuales parten del grafito. En esta investigación se usó cantidades mínimas de grafito para desprender una muestra capa por capa hasta llegar a una finísima capa de tal manera que se pueda analizar mediante un microscopio. Además, se busca incursionar y buscar elementos con los cuales se pueda realizar aleaciones, ya que el grafeno es considerado uno de los elementos del futuro ya que podría llegar a reemplazar a varios metales al ser capaz de formar estructuras ligeras quinientas veces más resistentes.

Palabras claves: Grafeno, Estructura Laminar, Anillos Hexagonales, Grafito

\section{Introducción.}

El grafeno se conoce desde la década de 1930, muchos investigadores han buscado llegar a tener un grano perfecto, ya que demuestra propiedades que puede contribuir de gran manera a todas las ramas de ingeniería, medicina en especial a toda la nanotecnología con la creación de nanomateriales a través del uso de carbón mineral. Al momento de descubrir un método de producción del grafeno la tecnología crecerá de manera increíble. Este elemento es una forma alotrópica del carbono, lo que determina al carbono es las distintas estructuras en las que se puede presentar desde el elemento más simple como es el grafito hasta el diamante. Este elemento se presenta en grandes cantidades dentro del cuerpo humano formando cadenas complejas, formando el $\mathrm{ADN}$ hasta ayudando a la producción grasas, el carbono se encuentra presente en muchos combustibles como combustible fósil, estos combustibles colaboran con importantes propiedades termodinámicas lo que les hace que estos tengan un gran poder calorífico.

El grafeno es un elemento novedoso ya que presenta una ordenación de átomos de carbono distinta en su estructura, formando una figura que se asemeja a un panal de abejas con formas onduladas, estos anillos se enlazan por una fuerza dipolar. Tiene un espesor de un átomo de carbono también conocido como monocapa de tal manera posee dos dimensiones: longitud, anchura; en si debe poseer tres dimensiones, pero la tercera corresponde a la altura, siendo esta altura que tiende a cero. Por otro lado, este material es muy estable pese a que su altura 
solo es del grosor de un átomo de carbono.

Figura 1. Estructura laminar del grafeno

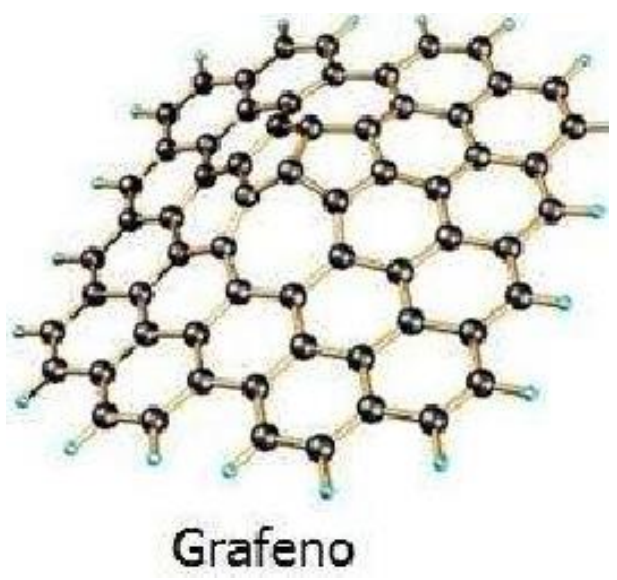

Los electrones del grafeno tienen un comportamiento distinto a cualquier material, así como otros materiales se rigen mediante ecuaciones. Este material se relaciona con el comportamiento de una partícula, es muy difícil detectar y generar. Para realizar este estudio es necesario un acelerador de partículas, utilizando un campo magnético para estimular partículas cargadas con electricidad de este modo las partículas empiezan a chocar y después alcanzar una velocidad extremadamente elevada. Estos equipos se pueden encontrar en otros países en laboratorios de investigación.

El uso de los metales en una gran parte han sido un problema por sus propiedades como el desgaste al paso de los años, deformaciones, mayor peso. En los vehículos puede ser usado el grafeno con resina epoxi como una aleación para ser elementos resistentes, ligeros y sin sufrir un mayor desgaste.

De la misma manera una capa de grafeno puede ser insertada en una capa de pintura por lo que los vehículos pueden resistir grandes colisiones o ser empleadas como aleaciones a metales ligeros como marco de la cabina de un vehículo, el grafeno es el futuro para crear tecnología favorable a nuestro bienestar.

El grafeno es un material ultraligero que busca incluirse en el mercado con el fin de proporcionar elementos estructurales livianos, termorresistentes. Por otro lado, este elemento puede sufrir aleaciones con otros materiales como cobre y níquel. El compuesto de cobre y grafeno es el que alcanza con 1,5 GPa., una resistencia 500 mayor que la del propio material por separado y un $50 \%$ más fuerte que el titanio, pero el segundo tampoco se queda atrás, al ser 200 veces más fuerte que el acero o tres veces más fuerte que las aleaciones de aluminio empleadas en construcción de estructuras. (Delgado, 2015)

El grafeno puede ser considerado como la base de distintas formas de carbono, considerada 
solo de dos dimensiones incluso si se juntan 3 millones de láminas del grafeno se lograría obtener un milímetro de grosor; si este material es envuelto en de manera arqueada en estructuras de cero dimensiones da como resultado fullerenos, mientras que si se realiza un dobles de la estructura de una dimensión se obtiene nanotubos, de la misma manera se puede apilar el grafeno en capas lo que daría como resultado el grafito como se puede encontrar en las puntas de los lápices formando una estructura en 3D como se puede observar en la figura 2. (Sandvik Coromant, 2017)

Figura 2. Distintas formas de carbono

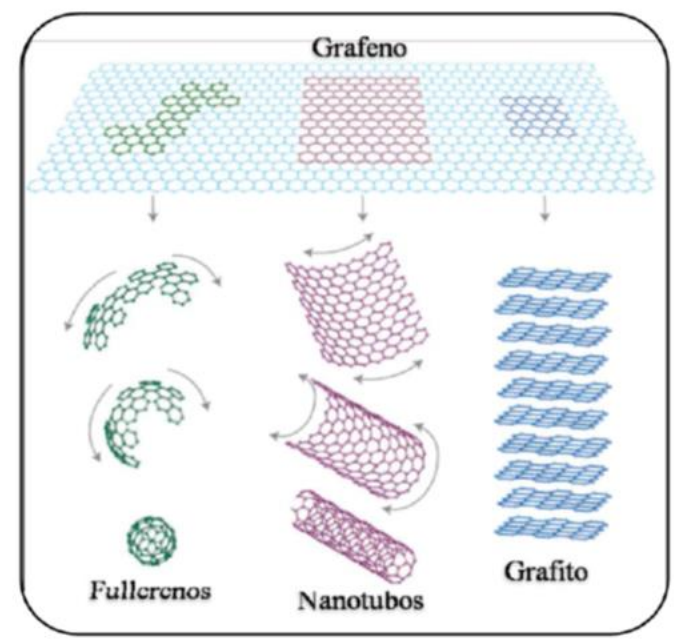

El grafeno se distingue por tres tipologías:

- Una capa (Monocapa)

- Dos capas (Dicapa)

- De tres a cuatro capas (Pocas capas)

- Intervalo de cinco a diez capas (Multicapa)

Las fuerzas con las que se unen estas capas son de suma importancia, aparte de la distribución, esto también denota al material, por ejemplo, el grafito tiene fuerzas muy fáciles de romper producto de tener un rozamiento con una superficie existe desgaste, por otro lado, el diamante es un material en el cual las capas están unidas por fuerzas muy grandes, esta configuración esta descrita por un enlace covalente en todas las direcciones. Por lo que si se desearía transformar el grafito de nuestro lápiz en un diamante se necesitaría una fuerza que supere 150000 veces la presión atmosférica. Sin embargo, varios investigadores buscan un método más sencillo para alcanzar la distribución del diamante. Es por esta razón que los diamantes se forman bajo tierra a temperaturas altísimas al igual que una presión elevada, es así que se forman los diamantes gracias a la colisión de las placas tectónicas.

Figura 3. Diferencia atómica del diamante y grafito 


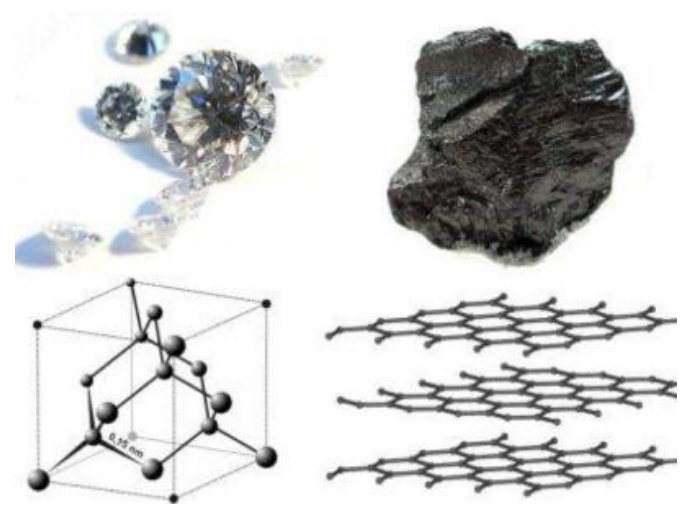

\section{Grafito}

El grafito es el montaje de distintas capas de carbono, muy finas que se entrelazan con facilidad, es decir, las fuerzas con las que actúan son muy débiles, hoy en día es usada en los lapiceros, la razón por la que se desgasta la punta del lápiz es porque estas capas de carbono se desprenden con facilidad. Cumple con una estructura cristalina de tal modo que se juntan de manera ordenada los átomos, este elemento se encuentra en tres dimensiones. (Quiroz Ceballos \& Hernández Gervacio, 2015)

En la mayor parte de lápices se usa el grafito para incorporar la punta en el fragmento de madera, se trata de una mina que está compuesta por carbono, arcilla y como producto de la cocción tenemos agua, estos son los ingredientes fundamentales de las puntas de los lápices comunes, entonces el carbono es el elemento que va a desprender un color gris con brillo mientras que la arcilla es el elemento que va a brindar dureza a la punta. (Orellana Gómez, 2018)

Figura 4. Porcentaje de arcilla en las minas de los lapiceros

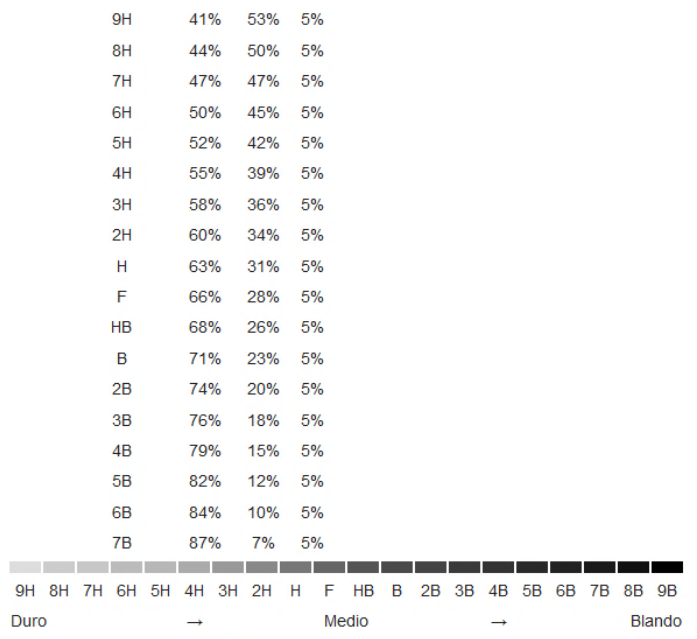




\section{Propiedades del grafeno}

Es necesario analizar sus propiedades para de mejor manera buscar las aplicaciones que este elemento puede tener ya sea grafeno puro o con aleaciones con metales ligeros, esta es la premisa para continuar con la investigación. Por la cantidad de propiedades que amerita este material el campo en el cual se le puede aplicar es muy amplio, con la ventaja de ser un elemento transparente y muy fino.

En su propiedad bidimensional, el grafeno está compuesto por una fina capa de un átomo de espesor, como esta medida es extremadamente pequeña solo se considera su existencia en dos dimensiones. Este elemento corresponde a una gran lista de elementos bidimensionales, pero tiene un comportamiento distinto a los otros. Si se apilan varias capas de grafeno se pueden lograr varias estructuras útiles para la tecnología.

El grafeno es ultraligero, ya que una lámina de grafeno de 1 metro cuadrado pesa solo 0,77 miligramos, lo que, en comparación con el acero (con la misma superficie), supondría un peso 200 veces menor. Este material consta con la capacidad de auto enfriado. En una aleación no tendría mayor repercusión ya que el peso no se podría distinguir en una balanza común. (Rodríguez González \& Oxana Vasilievna, 2014)

Es maleable, su constante elástica es enorme, tanto que una lámina de grafeno puede estirarse un $10 \%$ de su tamaño normal de forma reversible y puede doblarse hasta un $20 \%$ sin sufrir daño alguno. Al momento de realizar una aleación el material puede ubicar sus granos de manera uniforme por todo el material, cubriéndolo y aumentando su resistencia.

Es una de las propiedades que menor numero de investigaciones posee, gracias a pruebas de microscopia se ha logrado determinar valores numéricos que cuantifican las grandes presiones que este elemento puede resistir la resistencia a la tracción, $130 \mathrm{GPa}$, y el módulo de Young, 1 TPa que se encuentra entre los valores más altos de todos los materiales debido a un módulo de Young grande, el grafeno puede sufrir una gran elongación sin sufrir una ruptura, alcanzando una mayor pendiente en la gráfica de elongación. (Valencia, 2011)

Es resistente, este material es muy duro incluso más que el diamante, puede llegar a sustituir el acero estructural ya que el diamante es 100 veces más duro que el acero Tiene una resistencia mecánica de $42 \mathrm{~N} / \mathrm{m}$ (tensión de rotura), mientras que una lámina del acero más resistente y del mismo espesor que el grafeno, tendría aproximadamente una resistencia de $0,40 \mathrm{~N} / \mathrm{m}$, incluso el grafeno tiene una alta resistencia a ser rayado por lo que sería un excelente material para proteger una superficie. Varios estudios compilan que si se desea romper el material se necesita de un gran peso, aproximadamente cuatro toneladas.

Como su estructura está conformada por un espesor de un átomo de carbono, se forma una 
capa muy fina por lo cual se asemeja a un vidrio, es una de las razones por las que las empresas telefónicas desean ingresar con estas nuevas tecnologías además de tener flexibilidad, esta transparencia se debe a la velocidad con la que se mueven los electrones por toda la estructura brindando una mayor calidad. Incluso existen varias empresas que han encontrado muestras a nivel de laboratorio para iniciar con pantallas a base de grafeno.

Otra característica es la protección de la radiación, la ionización es el fenómeno en el que el átomo no llega a desprenderse del electrón, la estructura de anillos hexagonales empieza a sufrir una radiación por lo que los electrones devuelven sus estímulos, pero sin que estos se separen incluso cuando tienen como ejemplo una incidencia por parte de luz, nos ayuda a generar una reactividad química.

El campo eléctrico el grafeno tiene una forma alotrópica del carbono (el carbono por ser un elemento no metálico, es mal conductor de la electricidad) sin embargo este elemento presenta propiedades que corresponden a los metales, comportándose como semiconductor gap superficial o como semimetal de pequeño traslape, formando un campo eléctrico ambipolar o también conocido como tobera magnética, este término hace referencia al campo magnético convergente-divergente, es decir, guía, expande y acelera el estado de fluido similar a un gas o llamado plasma. (Rodríguez González \& Oxana Vasilievna, 2014)

Así mismo esta propiedad aplica a la multiplicación de frecuencias, la señal eléctrica atraviesa por el segmento por lo que la estructura del grafeno genera otra onda doble o triple, generando señales, pulsos, recepción de información a una velocidad increíble no comparada a la que tenemos en la actualidad.

La auto reparación de la capa es otra propiedad muy importante que tiene el grafeno, hace referencia al reemplazo de átomos por si algún átomo sufrió un desprendimiento total de la capa viene otro a sustituirlo de tal manera que la vida útil del material el cual sufrió la aleación aumenta de manera considerable, pero lo hará por un tiempo limitado, en comparación con otros metales el tiempo de durabilidad aumenta, representando una ventaja para el grafeno.

En el grafeno se pueden observar ondas que son dominadas onda de Dirac sin masa, pero no es tan simple de observar, es necesario confinarlas, se tienen registrado dos artículos científicos que han realizado dicha experimentación para lo cual se usa un punto cuántico para atrapar estas cuasipartículas, además es necesario tener un microscopio de efecto túnel, el punto cuántico es una unión p-n en el grafeno adoptando una forma circular, esto actúa como un potencial que mueve el nivel fermi por encima y por debajo de la energía asociada a los vértices de los conos de Dirac, fermi es el nivel de energía máximo.

Lo que hace el punto cuántico es dotas de un momento angular, cuando las cuasipartículas que tienen un gran momento angular colisionan se logran rebotes en el contorno, de este 
modo se logra de forma temporal atrapar las cuasipartículas. Al momento de atrapar la función se pueden observar nodos y antinodos con simetría circular, en este articulo hacen referencia a las ondas que son producidas al momento de botar una piedra en un rio. (Torres Silva \& López Bonilla, 2011)

Figura 5. Onda de los fermiones de Dirac
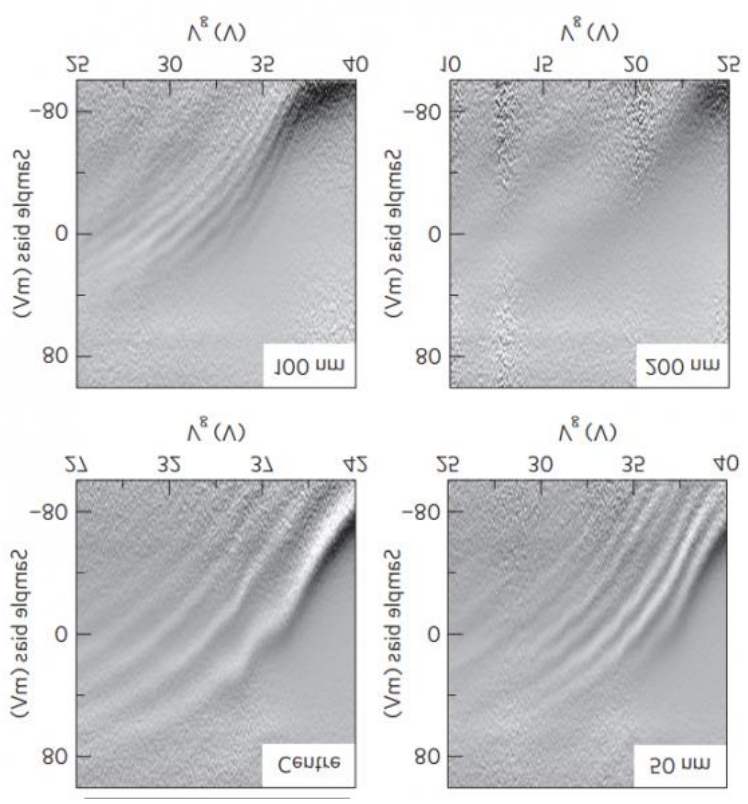

En el efecto Hall cuántico se ha observado en el grafeno a temperatura ambiente, los electrones dentro del grafeno se comportan como fermiones de Dirac sin masa. (Marquina, 2010)

\section{Tipos de grafeno}

El óxido de grafeno resulta de la oxidación y exfoliación de muchas capas de grafito, su nomenclatura es GO, además el oxido de grafeno tiene sus usos dependiendo del grado de oxidación que el grafeno contenga a continuación se presentan ciertas características: (Castro Beltrán, Sepúlveda Guzmán, De La Cruz Hernández, \& Cruz Silva, 2011)

- Hidrofílico, esta característica lo hace un elemento amigable con el agua y otros solventes orgánicos, al momento de combinarlos se pueden formar dos fases, la fase más grande que corresponde al liquido mientras que una fase pequeña hace referencia a pequeños granos sólidos.

- Gran área superficial.

- Capacidad de mezcla con polímeros y otros materiales.

- En capacidad eléctrica lamentablemente no se cumple como en el grafeno.

- Aplicando reducción, se puede bajar el oxígeno de este elemento y llegar al grafeno conocido como oxido de grafeno reducido. 
Para obtener grafeno es necesario reducir el oxígeno presente del grafito, al obtener el grafeno a partir de óxido de grafeno reducido, varias afirmaciones nos dicen que las propiedades si se cumplen, pero en la actualidad el óxido de grafeno reducido es muy difícil en su obtención además se necesita otros equipos muy costosos, por lo que se usa otro tipo de métodos para la obtención de grafeno.

\section{Aplicaciones}

El grafeno puede ser usado como conductor de electricidad en forma de pintura, varios investigadores han logrado combinar grafeno con un metal, de tal manera que al trazar una fina línea como si se tratara de un marcador esta puede conducir electricidad como si de un cable de cobre se tratase, incluso muchos elementos pueden ser montados sobre este segmento el cual les va a proporcionar energía ya sea solar almacenada o por una fuente de alimentación.

El grafeno se puede combinar con resina epoxi con el fin de crear plásticos mas ligeros y mas resistentes capaces de reemplazar a un metal, de esta manera se podrían crear piezas para ser usadas en automóviles, aviones, barcos, entre otras. La resina epoxi suele endurecerse cuando se combina con un catalizador o endurecedor en nuestro caso con el grafeno que hace referencia a un catalizador, tal como son los nanotubos de carbono. Incluso estos están tomando el lugar del platino en las pilas de hidrogeno, siendo esta una gran ventaja para usar un hidrolisis y de tal manera eliminar el motor de combustión interna, permitiendo que el hidrogeno impulse un vehículo. Lo que se busca es apilar una cantidad definida de capas de nanotubos. Se ha elegido este tipo de material ya que como esta formado por un solo átomo de espesor cualquier liquido o gas puede traspasar la capa sin sufrir ningún daño, caso contrario esto puede activar otras propiedades como la conducción eléctrica.

El grafeno puede ser usado para filtrar agua, es una gran ventaja ya que nuestro planeta tiene una cantidad elevada de agua salada, con lo cual una fina capa de grafeno permitiría que sea posible la desalinización. Es decir, que se elimina toda la sal con la que esta compuesta el agua de mar y transformarla en agua potable.

Existen varias empresas telefónicas que han buscado durante varios años el elemento que sea capaz de reemplazar las pantallas rígidas y frágiles usadas en los celulares, el grafeno es capaz de reemplazar este cristal, brindando al usuario un dispositivo ligero y resistente.

En el sector automovilístico se busca incursionar por aleaciones más resistentes y ligeras en especial por el chasis que es la base del automóvil, en especial por la seguridad de los ocupantes, si se logra un elemento aleado con el grafeno cuando exista una colisión las pérdidas humanas van a reducir de manera considerable, y no solo en los automóviles, en el campo de la mecánica estas aleación tendrían una gran aceptación, como se observó en las 
propiedades, la mayoría de estructuras podrían tener un recubrimiento de grafeno o una mezcla interna con otro elemento.

Este trabajo ha sido avalado por varios estudios, independientemente con un área respectiva de investigación en nuestro caso este estudio busca aleaciones con metales ligeros para verificar mediante un microscopio electrónico por lo cual es necesario tener sustentación acerca de las propiedades mecánicas que el grafeno posee, como también el análisis de estudios ya realizados. Nuestro enfoque no solo va orientado a la aleación con los metales, la resina epoxi con grafeno es una aleación con un gran potencial que podría dar un giro completo a la ingeniería.

Uno de los estudios más importantes es Measurement of the Elastic Properties and Intrinsic Strength of Monolayer Graphene por (Lee, 2008), esta investigación consiste en depositar membranas sin sufrir ningún proceso intrínseco sobre un sustrato de tal manera que se aplicaron cargas a través de la punta de un microscopio de AFM (Fuerza Atómica). Los experimentos arrojados en esta investigación muestran tanto un comportamiento elástico lineal como fractura frágil, de tal manera que este comportamiento es avalado por la siguiente ecuación que rige el grafeno ante cargas de tracción: (Lee, 2008)

$\sigma=E * \varepsilon+D * \varepsilon^{2}$

Al final de cada capa se pueden diferenciar dos tipos distintos de terminado de la estructura, no hay que confundir las propiedades que estos presentan ya que estas solo influyen en los anillos más cercanos a los bordes, se pueden encontrar dos estructuras como son en zigzag y armchair como se puede observar en la Figura 6, también se debería analizar los bordes como se realizo con el microscopio de fuerza atómica para verificar su resistencia en estos diferentes terminados.

Figura 6. Estructura del grafeno zigzag y grafeno armchair

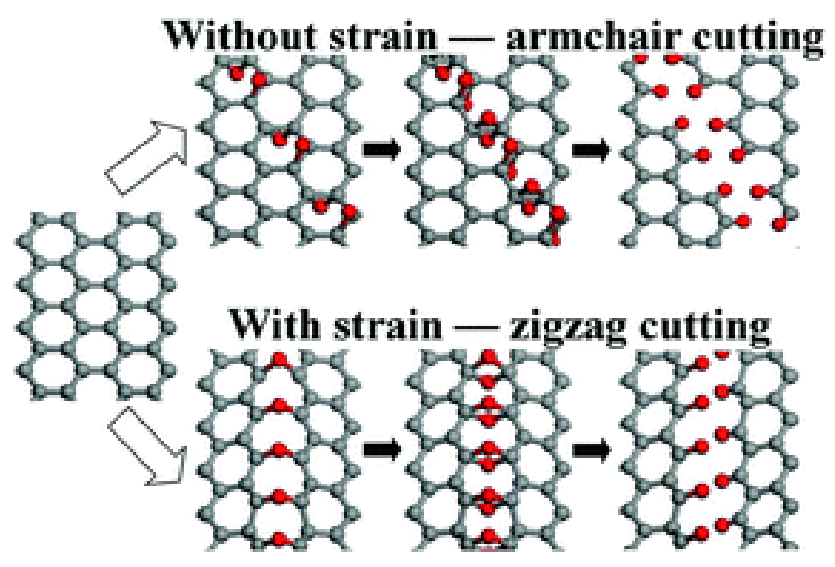

Además de haberse realizado varios estudios teóricos sobre el grafeno, muchos investigadores han buscado la forma más fácil de poder crear este compuesto sin necesidad 
de equipos sofisticados, en sí, estas obtenciones se las pueden realizar de forma solida o liquida, transformando grafito en grafeno.

Uno de los procesos más prometedores corresponde al grafeno que crece, hace referencia al crecimiento del grafeno en una superficie de metal en este caso se usa el níquel ya que en su estructura presenta átomos libres los cuales tejen una nueva estructura y este proceso se repite hasta extender esta capa. Como se muestra en la Figura 7, estas estructuras se enganchan formando una barrera tan fuerte difícil de separar.

Figura 7. Incrustaciones de grafeno en el níquel
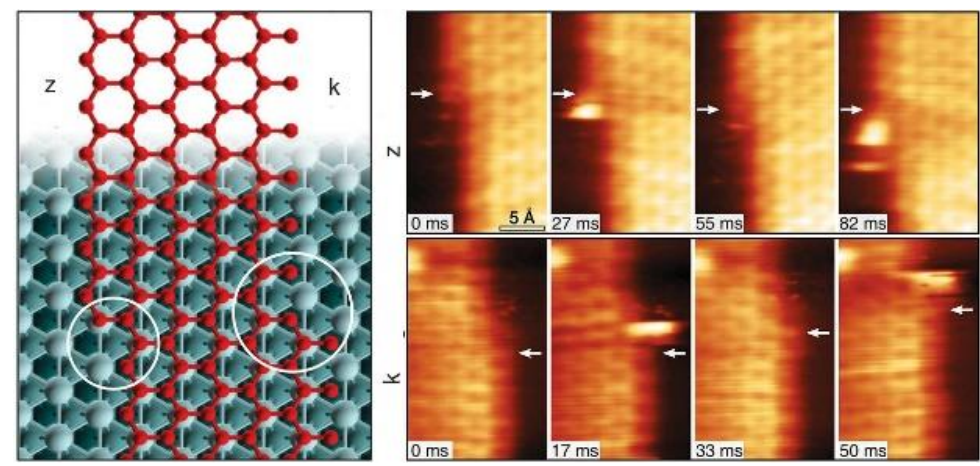

Se puede observar la estructura gris que corresponde al níquel y la estructura roja que corresponde al grafeno.

En la capa roja se pueden observar las estructuras de los bordes, en los círculos blancos son los puntos en los que se generan crecimiento de grafeno. El enganche se genera por la fuerza de la energía con la que giran los átomos libres de níquel, es lo que atrae a los átomos de carbono, de esta manera se logra extender la capa de grafeno, es necesario realizar varios estudios para conocer hasta que punto se va a producir este crecimiento, además se deben analizar otros metales que cumplan con el mismo comportamiento y saber si el grafeno actúa de la misma manera.

\section{Dislocaciones del grafeno}

El grafeno cumple con todas sus propiedades si su organización atómica es la correcta, pero siempre se van a encontrar anillos que no conservan su uniformidad, los defectos se dan en la primera dimensión también conocidos como defectos lineales. (Díez, 2014)

Figura 8. Dislocaciones del grafeno, desplazamiento de dipolos

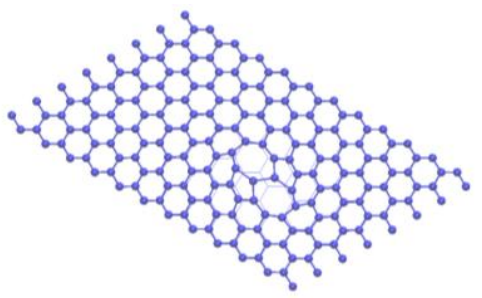


Estos defectos vienen de la mano con las longitudes de los dipolos, es decir, la aproximación de las moléculas polares. Cumpliendo la siguiente regla:

Se trata de una interacción atractiva cuando sus polos positivo y negativo se unen. La manera en la que ocurre cuando, la carga de una molécula polar es la que provoca la distorsión en la molécula apolar a través de su nube electrónica. (Guarnizo \& Martínez Yepes, 2001)

Hablamos de una interacción repulsiva cuando sus polos positivo y positivo se juntan o cuando sus polos negativo y negativo se aproximan. Por lo general en los líquidos y sólidos cumple con la interacción atractiva buscando una organización correcta.

Como se muestra en la Figura 8, los dipolos pueden influir en la estructura con su longitud, muchas veces se da este fenómeno por desplazamientos de ciertos átomos ubicados en esta finísima capa de grafeno, los anillos tienden a estirarse en una punta más que en la otra.

El espesor que este elemento posee tiende a cero, pero en esta aun influyen las leyes de la física, con su estructura se forma un gran campo magnético, velocidad elevada en sus electrones, transparencia, es esta la razón por la que se han realizado varios estudios en la Universidad de Manchester logrando verificar su estructura y propiedades como las que ya se describieron. Por su elevada velocidad se asemeja a las partículas elementales relativistas ya que cumple con dos reglas principales como una velocidad cercana a la luz, no interactúa con otros sistemas. (González, 2012)

Además, las cuasipartículas han sido estudiadas solo por dos artículos en Nature physics, en los cuales se pueden observar las gráficas de las ondas, como se resaltó en la función de onda de los fermiones de Dirac sin masa, en estos artículos fueron usados microscopios especiales para generar puntos cuánticos y para determinar las ondas. (Labra López, 2012)

\section{Importancia del problema}

El grafeno es un gran campo y se lo puede aplicar de distintas maneras, es una finísima capa de recubrimiento que se le puede emplear sobre un metal de tal manera que esta estructura puede absorber energía solar, y ser un excelente conductor de electricidad, y lo mejor es que una capa de grafeno no sufre en grandes cantidades el efecto Joule siendo una gran ventaja incluso para sustituir cables por una finísima línea que conduzca electricidad y varios elementos puedan ir montado en esta segmentación. (Kucinskis, Bajars, \& Kleperis, 2013)

El grafeno puede tener aleaciones con resina epoxi, es una gran manera de reemplazar los metales, como ya tuvo su explicación, esta aleación puede ser usada en lugar de piezas metálicas, la importancia de la estructura del grafeno como un catalizador ayuda al mercado de gran manera, aunque aún no existe un método de producción industrial se han podido 
verificar todas las propiedades ya antes dichas. Si se encuentra el método para una producción a gran escala, varias tecnologías se lograrán combinar siendo ahora menos costosas y mas eficientes, un ejemplo es la pila de combustible de hidrogeno, para sustituir un catalizador muy costoso como es el platino por nanotubos de carbono. (Méndez Medrano, Haret Codratian, \& Torres González, 2012)

Es de suma importancia continuar con la investigación, se busca formar un grano perfecto a través del grafito de tal manera que todas sus propiedades puedan ser explotadas al máximo y así revolucionar la industria, sustituyendo varios elementos que serían innecesarios al momento en que este grano se forme con gran exactitud. (Valverde Guijarro, 2014)

\section{Metodología}

La metodología que se va a usar para avalar nuestro artículo es una metodología experimental en la que se usa exfoliación mecánica para verificar una monocapa de grafeno, es uno de los métodos pioneros y sencillos de realizar sin necesidad de usar sofisticados aparatos de un laboratorio, lo que se busca con este método es desprender capa por capa de materia prima que en nuestro caso es el grafito. (Hernández Sampieri, Fernández Collado, \& Baptista Lucio, 2010)

Los materiales que se usan en la experimentación son:

- Una mina de lápiz 3H

- Una mina de lápiz HB

- Una mina de lápiz 2B

- Cinta Adhesiva

- Microscopio electrónico

- Placa de cobre (usada en el campo de electrónica para implementar circuitos)

El microscopio electrónico usualmente es usado en investigaciones por su gran poder de aumento en los lentes, superando la calidad de un microscopio óptico, además posee una resolución mayor, la forma en la que este dispositivo funciona es mediante el uso de electrones que son acelerados a gran velocidad, al momento en que estos electrones sufren el impacto se crea una luz para la iluminación, estos electrones son reflejados por la muestra o bien estos pueden atravesar, además estos equipos son capaces de analizar los compuestos de los cuales está formado la muestra que es expuesta.

\section{Técnica de elaboración}

Al momento de realizar la exfoliación mecánica se está cambiando la distribución, tan solo con la ayuda de una cinta adhesiva se desprenderá grafeno en polvo las veces que sea necesario hasta verificar que exista una capa tan fina, de tal manera verificar los resultados alojados por un microscopio electrónico alojado en las instalaciones de la Facultad de Mecánica ESPOCH, para verificar las capas usaremos distintos grados de dureza del grafito 
que se encuentra en las puntas de los lapiceros.

Como se explicó anteriormente la dureza que se generan en estas minas son gracias a la arcilla por lo que usaremos distintos tipos de dureza de un lápiz para verificar su estructura e identificar las diferencias y similitudes que existen en las capas.

Por otro lado, se va a realizar una exfoliación directa a una mina, es decir, vamos a fijar un trozo de cinta adhesiva a la mina compacta para desprender una capa fina, los resultados también serán avalados en el microscopio para verificar su estructura, se va a realizar esta prueba porque se supone que la mina solida tiene pequeñas partículas adheridas, lo que se presume es que van a salir muy separadas por la misma razón que no existe un desprendimiento constante como se lograría en la punta del lapicero.

La presente investigación está enfocada en analizar la estructura del grafeno usando las distintas técnicas que se explican en el apartado siguiente, para después lograr una combinación con resina epoxi de tal manera se necesitaran realizar pruebas para comprobar las propiedades ya explicadas. Por otro lado, se puede realizar recubrimientos de grafeno sobre metales ligeros, en si se busca reemplazar los elementos pesados por algo ligero y muy resistente, para mejorar el bienestar de nuestro entorno.

\section{Procedimiento}

Se desprende material de una mina de lápiz en manera de rayado en una hoja de papel para obtener una capa casi uniforme ya que si desprendemos grafito con una cuchilla se van a obtener granos muy grandes que en la cinta adhesiva no se va a poder separar de la mejor manera por lo que quedara montado un grano de grafito sobre la estructura de grafeno, al momento de realizar el rayado sobre el papel tomamos la cinta adhesiva para desprender el grafito del papel, se empieza a unir y desprender la mancha de grafito en distintos tramos de la cinta adhesiva hasta obtener la capa más fina de esta manera poder visualizarla en el microscopio electrónico, este procedimiento se va a realizar con las minas $\mathrm{HB} 3 \mathrm{H}$ y $2 \mathrm{~B}$.

Para la segunda técnica no es necesario desprender el grafito de la mina de lápiz tan solo vamos a impregnar un pedazo de cinta adhesiva, esta técnica no ha sido aplicada antes por lo que vamos a experimentar, al parecer en la mina solida solo existen una fina capa, en la cinta adhesiva solo se marca una pequeña mancha, los resultados que se esperan es una monocapa de grafeno. De la misma manera esta técnica se va a realizar en las tres distintas minas $3 \mathrm{H}$ HB y 2B, para después sacar semejanzas y diferencias con la técnica anterior, el microscopio electrónico es la herramienta fundamental para identificar la estructura del grafeno.

\section{Resultados}

Al momento de insertar la muestra al microscopio electrónico, como se observa en la figura 9, no se pudo distinguir ninguna diferencia, al momento de acercar y tener una mejor toma 
si se logró verificar unas pequeñas manchas sobre el cobre, para asegurarnos que las manchas eran de grafeno se encerró en un recuadro de color turquesa para hacer un análisis, el cual nos dio cobre y carbono como resultado, y se mostro como si se tratase de una aleación, en el recuadro de la derecha se detecta como una aleación, se puede observar una monocapa de grafeno, cuando se impregno la fina capa en cobre a simple vista no se pudo verificar ninguna diferencia, pero a nivel microscópico se observó finas capas.

Figura 9. Análisis de la muestra en el microscopio electrónico

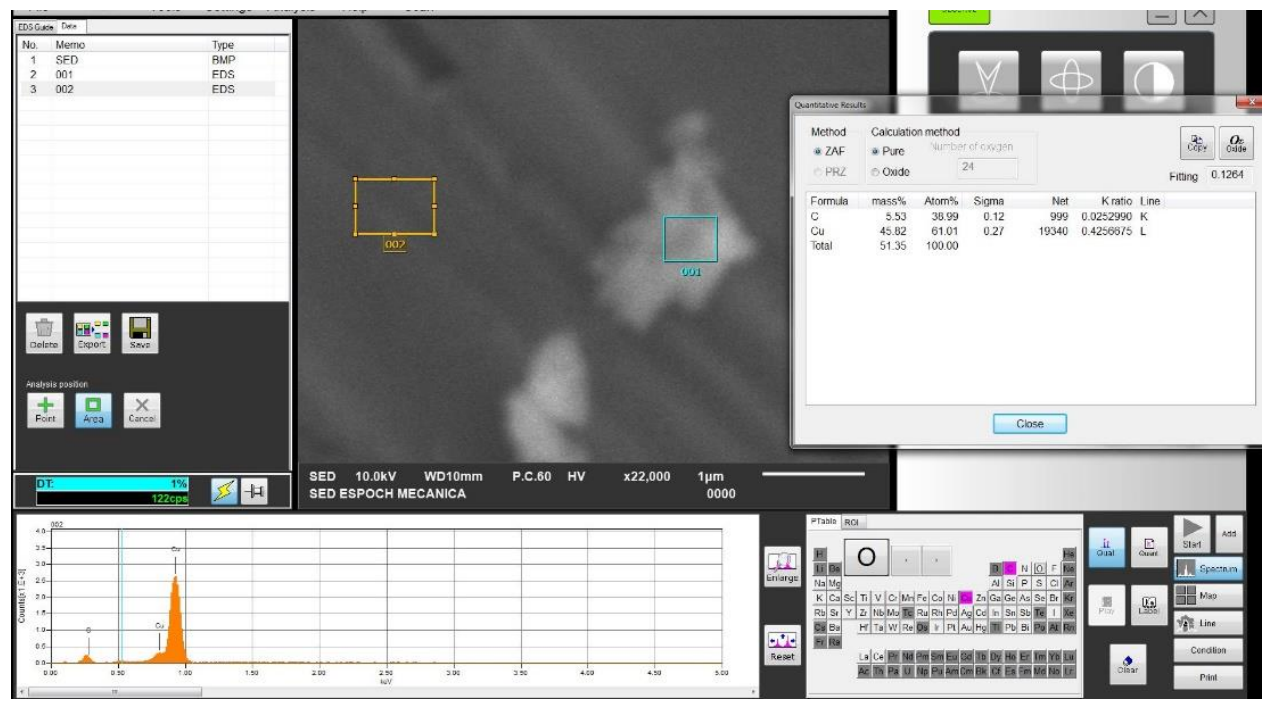

Como se observa en la figura10, al momento de mover la placa dentro del microscopio se encontraron varias muestras unas más finas que otras, en la placa de cobre existían grietas en las cuales pequeñas capas de grafeno ingresaban y se acoplaron de mejor manera como se puede observar en la capa del recuadro de color naranja la caja adopta la forma de las grietas de la placa mostrándose elástica.

Figura 10. Muestras con finas capas de grafeno

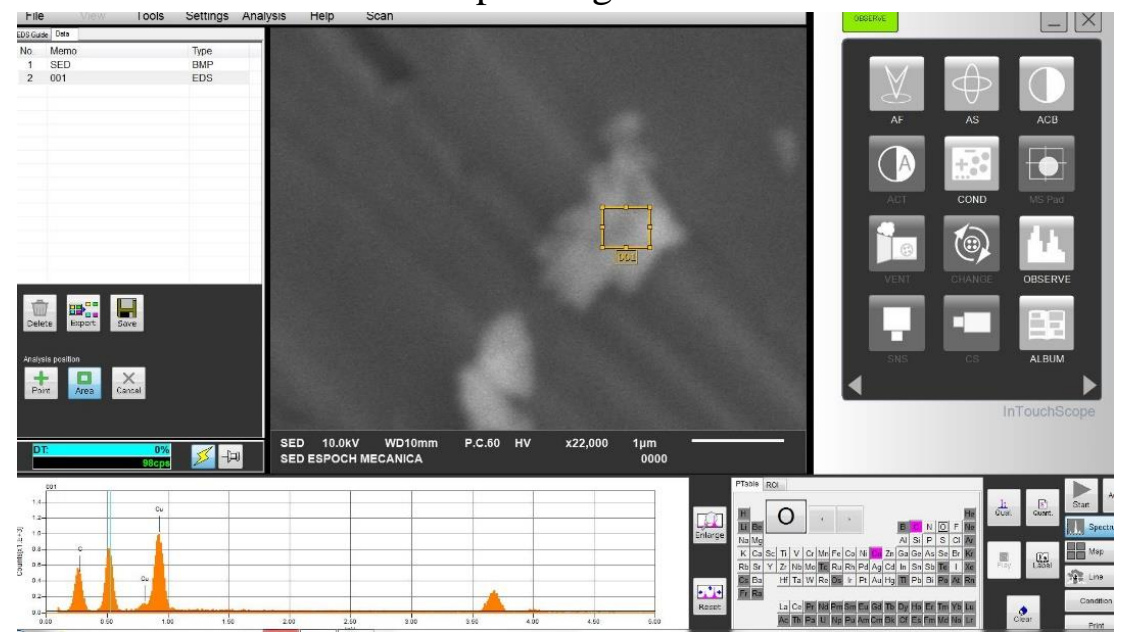

En la figura 11, se pueden verificar los distintos tamaños de las capas de grafeno mostradas 
en micrómetros, incluso existen pequeños puntos que no fueron medidos y se los observa tan delgados, lo ideal sería buscar un método para esparcir la capa de grafeno de la manera más uniforme, ya que por este método se verifican multicapas y lo primordial es encontrar una monocapa, además el grafeno debería cubrir una mayor área para realizar pruebas y verificar sus propiedades. Para cubrir una mayor área se debe realizar otro método de obtención del grafeno.

Figura 11. Tamaño de capas de grafeno

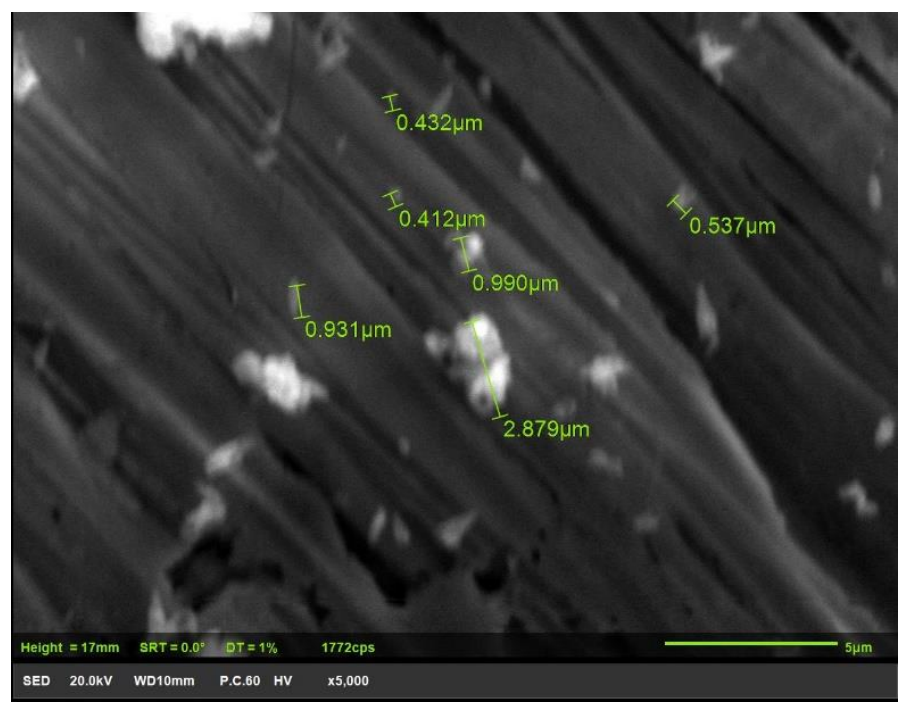

\section{Discusión}

Para realizar un mejor análisis a nivel estructural se necesita un equipo adecuado, en nuestro caso un microscopio con efecto túnel (Abril Bayón, 2017), nos sería de mucha ayuda de tal manera poner observar con el haz de luz la firma correspondiente a la capa de grafeno porque como se conoce, una monocapa bicapa obtienen una configuración distinta, de la misma manera podríamos usar este equipo para detectar las funciones de fermiones de dirac, ya que está investigación va dirigida al nivel estructural del grafeno. (Torres Silva \& López Bonilla, 2011)

Lo recomendable es realizar la experimentación con un lápiz de numeración $2 \mathrm{~B}$ porque como ya se explicó antes es necesario que el carbono que contenga en su estructura sea el más puro además al momento de rayar en el papel y adherirlo a la cinta se puede observar la mancha de mejor manera, mientras que si hacemos con el $3 \mathrm{H}$ el lápiz es muy oscuro y no se puede seguir las marcas en la cinta adhesiva.

Adherir y separar las capas de grafito de la manera más uniforme, aplastar con la misma fuerza en todas las secciones de la cinta ya que si no se realiza de esta manera no se podrá verificar capas uniformes y se verán multicapas de grafeno.

Para el uso del microscopio es necesario tener una placa de cobre pequeña en la cual se 
realicen varias pruebas y pueda girar la placa, ya que si la placa es muy grande y queremos analizar el otro extremo se debe apagar el equipo para girar, y esto demanda de mucho tiempo ya que no es un microscopio común.

Al momento de insertar al microscopio verificar la estructura de la cual se está analizando ya que las monocapa pueden ser confundidas con el material del cobre, al inicio de la experimentación se empezó a visualizar hexágono por lo cual se pensó que era grafeno hasta realizar la prueba de composición y nos dio como resultado cobre, después se movió la placa hasta encontrar estas manchas que eran el grafeno.

Buscar una zona en la cual la placa tenga un poco de imperfecciones para adherir la cinta en este lugar, presionar con fuerza en esta zona y desprender, entonces al momento de analizar en el microscopio electrónico se va a poder visualizar que el grafeno cumple con la propiedad de elasticidad, ya que se debe acoplar a la imperfección formando un recubrimiento.

Al momento de realizar este experimento es necesario usar guantes, y sacar las muestras en ese momento para que no se adhieran impurezas en la composición.

Se puede realizar otras investigaciones con otras formas de carbono para usar el método de exfoliación mecánica y de la misma manera llegar a otras conclusiones.

\section{Conclusiones.}

- Las pruebas realizadas con los distintos tipos de lápices fueron las mismas, no se encontró mayor diferencia todo está en la manera en la uniformidad con la que se desprende y adhiere las capas de grafito en la cinta adhesiva, ya que si se realiza un análisis de compuestos también nos dará carbono como resultado.

- Las pruebas realizadas sobre las minas no fueron tan efectivas ya que las capas de grafito encontradas fueron muy pocas, además se encontraron granos más grandes por la misma razón que al rayar en el papel se sufrió desprendimientos de grafito que quedaron sobre la mina.

- El grafeno en una futura investigación podrá tener una aleación con la resina epoxi ya que la manera en la que se acopla al material es efectiva como en este caso, se nota que es una capa elástica que adopta la forma en la que se le coloca, se busca un enfoque de aleación con la resina epoxi ya que de esta manera se podrán crear elementos resistentes, ligeros. Si la investigación y experimentación son efectivas esta aleación podrá reemplazar el metal.

- Si se encuentra oxígeno en el análisis de los compuestos es normal ya que las minas están expuestos al ambiente por lo que en los compuestos existe adherencia de oxígeno, en el microscopio electrónico solo se va a encontrar carbono, cobre, oxigeno. 


\section{Agradecimiento}

- El agradecimiento va dirigido a la Escuela Superior Politécnica de Chimborazo en especial a la Facultad de Mecánica por permitirnos realizar esta investigación con la ayuda del microscopio electrónico ubicado en los laboratorios de materiales, se logró sacar varias conclusiones que serán constructivas para futuras investigaciones con resina epoxi, y recubrimiento en otros metales para realizar pruebas, asegurando así las propiedades expuestas en este documento.

\section{Referencias bibliográficas.}

Abril Bayón, G. (2017). Aproximación continua al grafeno: El efecto túnel. Tesis de pregrado. Universidad de Valladolid. Facultad de Ciencias.

Arca Cebrián, F. (2016). Modelo de constantes de fuerza interatómicas. Bachelor Thesis. España: Mecánica de Medios Continuos y Teoría de Estructuras Escuela Técnica Superior de Ingeniería Universidad de Sevilla.

Askeland, D. (1998). Ciencia e Ingenieria de materiales. Mexico: International Thomson.

Castro Beltrán, A., Sepúlveda Guzmán, S., De La Cruz Hernández, W., \& Cruz Silva, R. (2011). Obtención de grafeno mediante la reducción química del óxido de grafito. Ingenierías, 34-42.

Delgado, R. A. (2015). Grafeno (Material del futuro). México: Instituto Tecnológico Superior Zacatecas Sur. Obtenido de silicon.es: https://www.silicon.es/al-finalinventaran-el-adamantium-compuesto-de-grafeno-500-veces-mas-resistente-que-laversion-original-112384

Díez, C. B. (2014). Modelo Discreto del Movimiento de Dislocaciones del Grafeno. Universidad de Sevilla, 58.

González, C. R. (2012). Obtención de hojas de óxido de grafeno para el desarrollo de nanocompositos poliméricos. Tesis de Doctorado. Universidad Autónoma de Nuevo León.

Guarnizo, F. A., \& Martínez Yepes, P. N. (2001). Experimentos de Química Orgánica. Eliscom Sas.

Hernández Sampieri, R., Fernández Collado, C., \& Baptista Lucio, P. (2010). Metodología de la investigación.

Kucinskis, G., Bajars, G., \& Kleperis, J. (2013). Graphene in lithium ion battery cathode materials: A review. Journal of Power Sources.

Labra López, L. (2012). Métodos de preparación de grafeno y derivados a partir de grafito y su incursión en la obtención de nanocompuestos polímero/grafeno. Tesis de especialidad. CENTRO DE INVESTIGACIÓN EN QU1MICA APLICADA.

Lee, C. (2008). Measurement of the Elastic Properties and Intrinsic Strength of Monolayer Graphene. Pubmed, 388. 
Marquina. (2010). Espectroscopía Raman del grafeno monocapa y el grafito: acoplamiento electron fonon y efectos no adiabaticos. Tumbaga, 194.

Méndez Medrano, M., Haret Codratian, R., \& Torres González, L. (2012). Grafeno: el alótropo más prometedor del carbono. Acta Universitaria.

Orellana Gómez, C. A. (2018). Estudio de métodos alternativos de producción de grafeno . Tesis Doctoral. UNIVERSIDAD TECNICA FEDERICO SANTA MARÍA.

Quiroz Ceballos, D. M., \& Hernández Gervacio, C. (2015). Grafeno: Estado del arte. Tesis de maestría. CENTRO DE INVESTIGACIÓN EN MATERIALES AVANZADOS.

Rodríguez González, C., \& Oxana Vasilievna, K. (2014). Propiedades y aplicaciones del grafeno.

Sandvik Coromant. (2017). Grafeno: biografía de un material . bachelor Thesis. Universidad Nacional de Educación a Distancia (España). Facultad de Filosofía . Obtenido de sandvik.coramant.com: https://www.sandvik.coromant.com/eses/services/engineering/stories/pages/a-material-that-may-change-the-world.aspx

Torres Silva, H., \& López Bonilla, J. (30 de junio de 2011). Aspectos quirales del grafeno. Ingeniare, 67-75.

Valencia, G. A. (14 de Abril de 2011). El Grafeno. Revista Colombiana de Materiales.

Valverde Guijarro, Á. L. (2014). Estudio de las propiedades del grafeno y sus posibilidades.

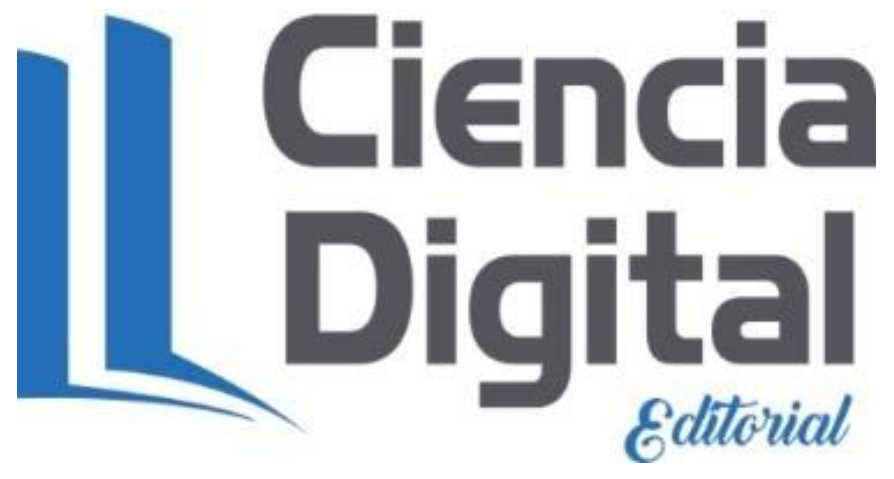




\section{Para citar el artículo indexado.}

Guacho E., Padilla C., Buenaño L. \& Cuaical B. (2019). Obtención de capas de grafeno a través de la exfoliación mecánica del grafito. Revista electrónica Ciencia Digital 3(1), 313332. Recuperado desde:

http://cienciadigital.org/revistacienciadigital2/index.php/CienciaDigital/article/view/294/70 $\underline{0}$

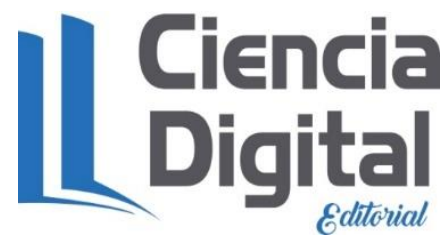

El artículo que se publica es de exclusiva responsabilidad de los autores y no necesariamente reflejan el pensamiento de la Revista Ciencia Digital.

El artículo queda en propiedad de la revista y, por tanto, su publicación parcial y/o total en otro medio tiene que ser autorizado por el director de la Revista Ciencia Digital.
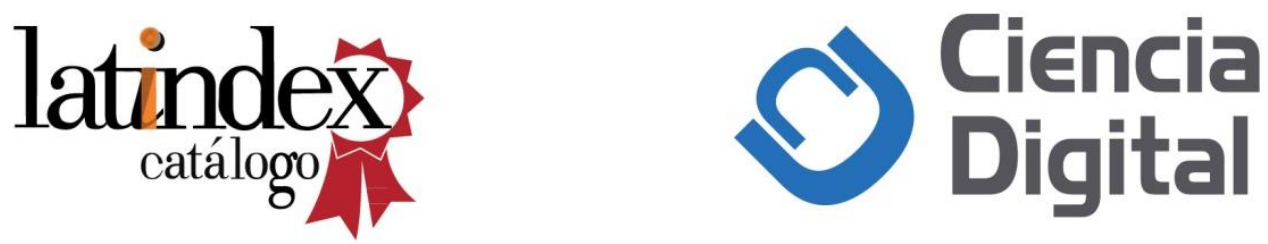